\title{
Editorials
}

\section{Better care through better use of data in GP-patient partnerships}

In 1988 Julian Tudor Hart prescribed 'A new kind of doctor', calling for data-intensive, community-responsive primary care. ${ }^{1} \mathrm{He}$ argued for a realignment of primary care with the needs of populations rather than individuals; and for greater emphasis on prevention. ${ }^{1}$ These principles are largely ingrained in modern UK general practice: clinical commissioning groups (CCGs), the Quality and Outcomes Framework (QOF), and audit all require primary care to consider information about practice populations; and disease prevention is routine practice. The new era of 'big data' is likely to escalate further such approaches but may also change the conversation of primary care between patients, practitioners, and the public.

Systematic collection, collation, and analysis of data were core to Hart's manifesto. Today's primary care has more advanced tools at its disposal. Electronic health records are the foundations on which we build alerts and reminders to guide decisions at the point of care. Electronic templates help capture key data on conditions and care pathways. These data can be extracted across IT systems for research (for example, the Clinical Practice Research Datalink [CPRD], The Health Improvement Network [THIN], QResearch, and ResearchOne in the UK; the NIVEL Primary Care Database in the Netherlands; or SIDIAP in Spain), and across populations to support service development (for example, audit, uww.openprescribing. net) and incentivise activity (for example, QOF in the UK, the Primary Health Organization [PHO] Performance Programme in New Zealand, or Practice Incentives Program [PIP] in Australia].

Traditionally, large-scale data extracts of NHS care records have been seen as the way to realise the more systematic primary care that Hart envisioned. Extracted records may be combined with other data at a national level such as hospital admissions and discharges from claims data, or official government statistics such as death registrations. More detailed information at regional level, however, is more complex, especially across important boundaries such as between health and social care. This is unsurprising considering each local health system may host vast quantities of databases that record and support care. ${ }^{2}$ Also, the traditional paradigm of core minimum datasets is being challenged because multiple aspects of our daily lives are now linked by common technologies such as smartphones and smartwatches generating health-relevant data invisibly, like patterns of movement, and interactively, like apps for symptom monitoring, medication reminders, and access to primary care records. The potential to link primary care with the digital by-products of everyday life holds the promise of better prevention, selfcare, and monitoring. But the community and population focus of primary care could be weakened through inequalities: the better off and the healthy may well find it easier to engage with the digital age.

\section{TWO NEW KINDS OF DATA}

We see two main types of new 'big data' impacting on primary care in future: active and passive. Active in situations where the user is consciously producing health-related data, for example, by interacting with apps, and passive where they are not, for example, accelerometer data from smartphones or smartwatches.

There is a plethora of apps already available, though relatively few that have been validated to provide credible data. The UK NHS is currently building a library of 'approved' apps, although the number of apps being developed is likely to outstrip the capacity for regulation and accreditation in the UK or other countries. Consequently, some apps, which are being used for both clinical research and care, have developed their own validation paths. In the UK, ClinTouch (www.clintouch.com) uses validated questions and methods to support patients with psychosis to monitor their symptoms, and alert their clinical team if a problem is suspected; Cloudy with a Chance of Pain (mww.cloudywithachanceofpain.com) collects symptom data from patients with arthritis directly from their smartphone to investigate the association with weather PatientView (www.patientview.org) is a website that enables patients with kidney disease, inflammatory bowel disease, or diabetes to view their medical records, and add symptoms and patient-reported outcomes. Internationally, Singapore's Ministry of Health provides a range of webbased and smartphone apps for patients (wnw.healthhub.sg), and in primary care, Apple Health enables patients to access their electronic medical record and input physiological measurements (compatible with various different vendors). Apps can also provide evidence-based treatments recommended by clinical guidelines, such as cognitive behavioural therapy (CBT), which provide data on usage and adherence.

By contrast, passive data may be collected from a variety of sources. Smart electricity meters collect data that might be used to predict whether older patients have fallen or have a change in daily living pattern indicating they have run into problems. ${ }^{4}$ Studies from the US demonstrate that analysis of social media may identify symptoms related to disease outbreaks ${ }^{5}$ or mental health problems. ${ }^{6}$ Location technologies in mobile phones can track patients with dementia and alert health services if they are in danger. Smartwatches can detect seizures or characterise tremors. ${ }^{7}$ Dosette boxes linked to the internet can provide useful insights into medication adherence. Everyday life is becoming routinely digital.

The primary care record could form a vital bridge between the active and passive data sources above - creating new insights for individual patient care, population care and research. For example, analysing a patient's home blood pressure readings may help identify white-coat hypertension and avoid unnecessary increases in medication assessing a patient with depression's adherence to smartphone-based CBT may identify alternative treatments; and knowing that a patient has not opened their dosette box of hypoglycaemic medications could explain their uncontrolled diabetes. Understanding changes in population patterns of physical activity could bridge primary care and public health approaches to health improvement. Trends in social media content and web searches for symptoms signal disease outbreaks and could help with immediate service planning. For research, more deeply connected data across multiple, bettercharacterised populations can transform (clinical) epidemiology and feed evidence deserts such as understanding the needs of patients with multiple conditions.

\section{THREE BIG CHALLENGES}

Big data, however, bring big challenges including: governance, evaluation, and unintended consequences. The governance regarding 'active' data sources is relatively straightforward, as users can give consent and see what the data are being used for and by whom. If these data are used for 
unintended or unclear purposes, users can be made aware and objections subsequently raised. 'Passive' data are more problematic as users may not be aware they are generating health-related information. Dame Fiona Caldicott's latest report asks us to reconsider the nature of consent and patient feedback on data uses. ${ }^{8}$ Possibilities include dynamic consent, whereby patients control consent continuously and receive information about the uses of their data. These challenges are not unique to medicine though. There is a general move towards citizens controlling their own data, and being able to see how their data are used across the spectrum of public and private services. The UK Department of Health's new Connected Health Cities initiative reuses wider civic digital governance for better health data analytics (www.connectedhealthcities.org).

The second challenge is that new data sources require rigorous evaluation before introduction. For example, to employ smart electric meter data requires evidence that has not yet been generated. ${ }^{4}$ Telecare research may or may not be relevant. ${ }^{10}$ These are complex interventions where implementation and evaluation are difficult, ${ }^{11}$ but to ignore available and potentially valuable data that may improve care is arguably negligent. On the other hand, digital interventions have come unstuck where they are rolled out without due evaluation and understanding, such as the Summary Care Record in the UK's out-of-hours care. ${ }^{12}$ A major challenge for vendors of clinical information systems is to avoid data corruption or security breaches through linked apps, therefore very few apps are likely to be approved for connection.

Third is the digital cousin of "primum non nocere' (first do no harm), and the potential unintended consequences of introducing new 'big' data sources. An immediate risk from the explosion in connected health technologies is to exacerbate the 'digital divide' where the under-served are under-sampled - a modern equivalent of Hart's inverse care law. Older and poorer people may become more isolated as healthcare depends on consumption of domestic technologies. This would also impact on population health management and research, where data samples are not representative of the population being served. A greater emphasis on ubiquitous over 'nice to have' consumer technologies is one way to mitigate this risk. Workload inflation is another potential problem - a blizzard of poorly or partially analysed data will further stretch primary care resources, which are already under considerable pressure. ${ }^{13}$ For example, daily blood pressure recordings sent via apps are not useful in every patient but still require processing, risking overwhelming clinicians. Systems should be developed that only convey essential information, and any additional workload should be adequately resourced. Furthermore, a lack of preparedness for more clinical data may precipitate over-reaction, for example, unnecessary hospitalisation when a patient with COPD's oxygen saturations sent via telecare benignly dip. While in the research setting, more linked data may tempt researchers to 'overfit' and report specious associations.

\section{CONCLUSION}

In a more connected world, there are new opportunities to link data to primary care records that go beyond the traditional paradigm of 'big' health data. The UK is ideally placed to reveal important new understanding about the interactions of biology, behaviours, and environments and primary care is at the heart of that nexus. Like Hart, we see no dividing line between research and care quality improvement, and therefore call for an honest conversation with patients and citizens about the value of the data they generate. This should build on the strong traditions of using primary care data for these purposes, but should also evolve to connect with community-based data sources that can provide a bigger picture of health and care. However, these new opportunities also pose big challenges, and careful evaluation and negation of unintended risks are vital.

\section{Benjamin Brown,}

GP and Wellcome Trust Research Training Fellow, Health eResearch Centre, Farr Institute of Health Informatics Research, Centre for Health Informatics, University of Manchester, Manchester.

\section{Liam Smeeth,}

Professor of Clinical Epidemiology, London School of Hygiene and Tropical Medicine, London.

\section{Tjeerd van Staa,}

Professor of Health eResearch, Health eResearch Centre, Farr Institute of Health Informatics Research, Centre for Health Informatics, University of Manchester, Manchester.

\section{lain Buchan,}

Professor of Public Health Informatics, Health eResearch Centre, Farr Institute of Health Informatics Research, Centre for Health Informatics, University of Manchester, Manchester.

\section{Competing interests}

The authors have declared no competing interests.

\section{ADDRESS FOR CORRESPONDENCE}

\section{Benjamin Brown}

Health eResearch Centre, Farr Institute of Health Informatics Research, Centre for Health Informatics, University of Manchester, Vaughan House, Portsmouth Street, Manchester M13 9PL, UK

E-mail: benjamin.brownamanchester.ac.uk

\section{Provenance}

Commissioned; externally peer reviewed.

DOI: https://doi.org/10.3399/bjgp17X688921

\section{REFERENCES}

1. Hart JT. A new kind of doctor: the general practitioner's part in the health of the community. London: Merlin Press, 1988.

2. Ainsworth J, Buchan I. Combining health data uses to ignite health system learning. Methods Inf Med 2015; 54(6): 479-487.

3. Kinderman $P$, Hagan $P$, King $S$, et al. The feasibility and effectiveness of Catch It, an innovative CBT smartphone app. BJPsych Open 2016; 2(3): 204-209.

4. Liu L, Stroulia E, Nikolaidis I, et al. Smart homes and home health monitoring technologies for older adults: a systematic review. Int J Med Inform 2016; 91: 44-59.

5. Nagar R, Yuan Q, Freifeld CC, et al. A case study of the New York City 2012-2013 influenza season with daily geocoded Twitter data from temporal and spatiotemporal perspectives. $J$ Med Internet Res 2014; 16(10): e236.

6. McManus K, Mallory EK, Goldfeder RL, et al. Mining Twitter data to improve detection of schizophrenia. AMIA Jt Summits Transl Sci Proc 2015; Mar 25: 122-126.

7. Reeder B, David A. Health at hand: a systematic review of smart watch uses for health and wellness. J Biomed Inform 2016; 63: 269-276.

8. National Data Guardian for Health and Care. Review of data security, consent and opt-outs. London: National Data Guardian, 2016.

9. Williams H, Spencer K, Sanders C, et al. Dynamic consent: a possible solution to improve patient confidence and trust in how electronic patient records are used in medical research. JMIR Med Inform 2015; 3(1): e3.

10. Henderson C, Knapp M, Fernández JL, et al. Cost effectiveness of telehealth for patients with long term conditions (Whole Systems Demonstrator telehealth questionnaire study): nested economic evaluation in a pragmatic, cluster randomised controlled trial. BMJ 2013; 346: $f 1035$

11. Moore G, Audrey S, Barker M, et al. Process evaluation of complex interventions: UK Medical Research Council (MRC) guidance. 2014.

12. Greenhalgh T, Stramer K, Bratan T, et al. The devil's in the detail: final report of the independent evaluation of the Summary Care Record and HealthSpace programmes. London: University College London, 2010.

13. Majeed A. Primary care: a fading jewel in the NHS crown. London J Prim Care (Abingdon) 2015; 7(5): 89-91. 